\title{
Constraints on the Profiles of Total Water PDF in AGCMs from AIRS and a High-Resolution Model
}

\author{
ANDREA MOLOD* \\ Global Modeling and Assimilation Office, NASA Goddard Space Flight Center, Greenbelt, Maryland
}

(Manuscript received 29 July 2011, in final form 21 June 2012)

\begin{abstract}
Atmospheric general circulation model (AGCM) cloud parameterizations generally include an assumption about the subgrid-scale probability distribution function (PDF) of total water and its vertical profile. In the present study, the Atmospheric Infrared Sounder (AIRS) monthly-mean cloud amount and relative humidity fields are used to compute a proxy for the second moment of an AGCM total water PDF called the "RH01 diagnostic," which is the AIRS mean relative humidity for cloud fractions of 0.1 or less. The dependence of the second moment on horizontal grid resolution is analyzed using results from a high-resolution global model simulation.

The AIRS-derived RH01 diagnostic is generally larger near the surface than aloft, indicating a narrower PDF near the surface, and varies with the type of underlying surface. High-resolution model results show that the vertical structure of profiles of the AGCM PDF second moment is unchanged as the grid resolution changes from 200 to 100 to $50 \mathrm{~km}$, and that the second-moment profiles shift toward higher values with decreasing grid spacing.

Several Goddard Earth Observing System, version 5 (GEOS-5), AGCM simulations were performed with several choices for the profile of the PDF second moment. The resulting cloud and relative humidity fields were shown to be quite sensitive to the prescribed profile, and the use of a profile based on the AIRS-derived proxy results in improvements relative to observational estimates. The AIRS-guided total water PDF profiles, including their dependence on underlying surface type and on horizontal resolution, have been implemented in the version of the GEOS-5 AGCM used for publicly released simulations.
\end{abstract}

\section{Introduction and motivation}

Cloud processes in the atmosphere have an important influence on climate and climate change, and therefore must be accurately depicted in global climate and weather models. Many of the cloud processes occur at scales that are not resolved by even the highest-resolution models currently in use, and therefore must be parameterized. As part of any atmospheric general circulation model (AGCM) parameterization of cloud processes, such as condensation and evaporation of cloud water and precipitation and the analogous processes for frozen

\footnotetext{
* Additional affilliation: Earth System Science Interdisciplinary Center, University of Maryland, College Park, College Park, Maryland.
}

Corresponding author address: Andrea Molod, Earth System Science Interdisciplinary Center, University of Maryland, College Park, 5825 University Research Court, Suite 4001, College Park, MD 20740.

E-mail: andrea.m.molod@nasa.gov condensate, an assumption must be made about the subgrid-scale distribution of total water or a related quantity. These assumptions can range from an "on-off" approach to the computation of condensation/sublimation in a grid box to the use of multiparameter prognostic probability distribution functions (PDFs) of total water.

The approach used in many current AGCMs (e.g., Schmidt et al. 2006; Neale et al. 2010; Donner et al. 2011; Collins et al. 2008) is to assume that total water in a grid box is distributed according to a simple two-parameter symmetric probability distribution function such as a "top hat" (e.g., Bacmeister et al. 2006; Tiedtke 1993) or "triangular" distribution (e.g., Smith 1990). The two parameters of the distribution are related to the first two moments of total water. Studies have also been performed with implementations of probability distributions with more parameters, such as an asymmetric triangular PDF (Watanabe et al. 2009) or a beta PDF (Tompkins 2002).

Estimates of the parameters for the different PDFs either in current use or proposed for use are obtained in several ways. Slingo and Ritter (1985), for example, used a PDF second moment that varies in height only, and 
was chosen to minimize errors in the AGCM-simulated radiative fluxes. Choosing the PDF second moment in this manner essentially involves trial and error, in an attempt to match AGCM-simulated surface and top-ofatmosphere radiative fluxes with existing observational estimates. Many current AGCM PDF parameter choices and their variation with height are also chosen in this manner (Neale et al. 2010; Schmidt et al. 2006; Collins et al. 2008). In other studies, Tompkins (2002) chose the PDF shape and parameters from the results of cloud-resolving model (CRM) simulations and developed a prognostic computation of the PDF moments, and Zhu and Zuidema (2009) concluded from their analysis of in situ data and high-resolution model simulations that joint PDFs of water, thermodynamic, and dynamic variables were needed to predict cloud amount in the boundary layer. A detailed description of many of the various PDF schemes in current use or proposed is presented in Tompkins (2002).

An observational computation or guideline for the AGCM total water PDF parameters is desirable, but none of the techniques used to estimate the total water PDF parameters for an AGCM use in situ or satellite measurements. A technique has been studied by Norris et al. (2010) to directly compute the subgrid-scale variability of cloud water using data from the Moderate Resolution Imaging Spectroradiometer (MODIS), but MODIS cannot provide information about the vertical dependence of the PDF parameters needed by AGCM parameterizations of moist processes.

An approach is presented here for using data from the Atmospheric Infrared Sounder (AIRS) on board the Aqua spacecraft to compute a proxy for the width of an AGCM total water PDF and its variation with height. The next section contains the details of the computation of the proxy for the PDF's second moment, a demonstration of the correspondence between the proxy and the second moment itself, and a description of the behavior of the proxy. In section 3 the issue of the dependence of the PDF second moment on the AGCM horizontal grid resolution is addressed using results from a global highresolution simulation, and in section 4 results from AGCM simulations using the AIRS-guided PDF parameters are shown. The final section contains a summary of the current findings and a discussion of some future work to expand on the AGCM parameterization.

\section{RH01 diagnostic: Proxy for the second moment of the total water PDF}

\section{a. PDF width and critical relative humidity}

For any given probability distribution function of total water, the cloud fraction $C_{f}$ and total condensate $q_{c}$ can be written as

$$
\begin{aligned}
& C_{f}=\int_{q^{*}}^{\mathrm{inf}} P\left(q_{T}\right) d q_{T}, \text { where } \int_{-\mathrm{inf}}^{\mathrm{inf}} P\left(q_{T}\right) d q_{T}=1, \\
& \text { and } q_{c}=\int_{q^{*}}^{\mathrm{inf}}\left(q^{*}-\overline{q_{T}}\right) P\left(q_{T}\right) d q_{T} .
\end{aligned}
$$

Here $q^{*}$ is the saturation specific humidity for the grid box, $q_{T}$ is the total water, $P\left(q_{T}\right)$ is the probability of finding a particular value of $q_{T}$ in a grid box, the overbar denotes the gridbox mean value, and horizontal variations of temperature are considered to be small. The equations state that condensation occurs for the values of $q_{T}$ that exceed saturation, and that the cloud fraction is the fraction of the grid box where $q_{T}$ exceeds saturation. The assumption that the temperature fluctuations are small is discussed in Tompkins (2002), where he suggests an approach for considering the temperature fluctuations but cites an observational study that indicates that to zeroth order they may be neglected.

The clearly demarked end of a discrete PDF results in a clear relationship between the width of the PDF and a "critical relative humidity" $\left(\mathrm{RH}_{c}\right)$, which is the AGCM gridbox relative humidity at which condensation or deposition begins; $\mathrm{RH}_{c}$ is also a limiting relative humidity for the evaporation and sublimation processes, which are restricted to prevent the gridbox relative humidity from exceeding $\mathrm{RH}_{c}$. The threshold behavior is illustrated schematically in Fig. 1. The solid black rectangle represents a top hat-shaped PDF of total water, with $\overline{q_{T}}$ and width $\sigma$. The value of the gridbox saturation specific humidity is marked as $q^{*}$, and the shaded area is the amount of condensate, which, according to the above-mentioned equation, is the integral under the PDF from $q^{*}$ to infinity. The blue rectangle in the figure represents a PDF with the same mean but a smaller value of $\sigma$. For this PDF the integral under the PDF from $q^{*}$ to infinity is zero, and the AGCM algorithm will not produce any new condensation. The red rectangle in the figure depicts the narrowest possible PDF, for which the condensation would only take place if $\overline{q_{T}}$ is equal to or greater than the saturation specific humidity. For the top hat PDF depicted in Fig. 1, the relationship between $\sigma$ and the critical condition for condensation can be expressed as

$q^{*} \leq \overline{q_{T}}+\sigma q^{*} \rightarrow \frac{q_{T}}{q^{*}}=1-\sigma \rightarrow \mathrm{RH}_{\text {crit }} \equiv 1-\sigma$.

For continuous PDFs, such as a Gaussian, the width of the PDF can be defined as one or two (or more) standard deviations from the mean, and therefore the critical relative humidity is not uniquely related to the PDF second moment. However, there still exists an analogous 


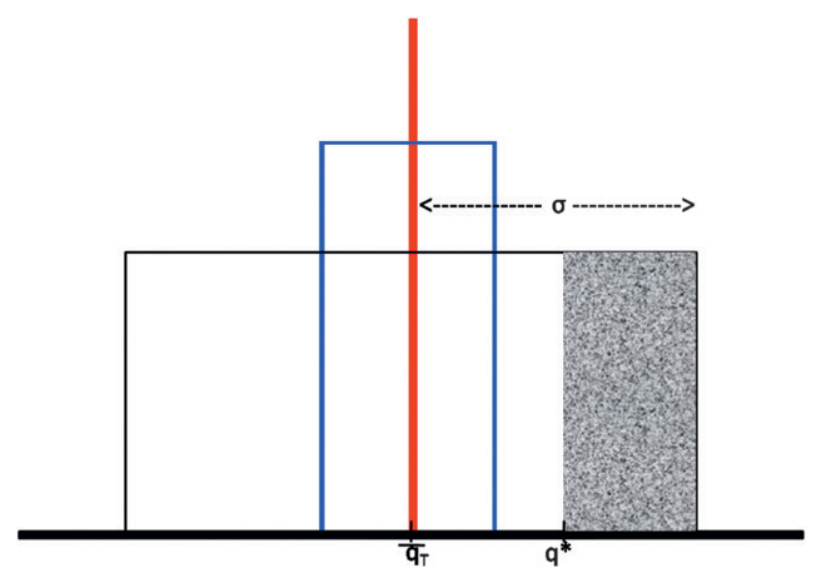

FIG. 1. Schematic of a "top hat" PDF for $q_{T}$. The shaded area represents the amount of condensation when the distribution is wide enough for condensation (black line). The blue line represents a narrower distribution for which there would be no condensation in this grid box.

relationship between the width of the distribution and the gridbox mean $\mathrm{RH}_{c}$.

\section{b. AIRS data and the definition of the "RHO1 diagnostic"}

The AGCM conceptualization of $\mathrm{RH}_{c}$ was defined in the previous section as the gridbox relative humidity at which condensation or deposition begins and to which evaporation and sublimation may proceed. The cloud cover in an atmosphere near this critical value is therefore expected to be small. A proxy for the critical relative humidity may therefore be computed by sampling the atmospheric relative humidity in grid boxes with "newly formed" cloud, or grid boxes with cloud fractions that are small. A diagnostic called "RH01" is defined here as the relative humidity averaged over grid boxes where the cloud fraction is less than $10 \%$, that is,

$$
\begin{aligned}
& \mathrm{RH} 01 \equiv \mathrm{RH} \text { of grid boxes with } \\
& 0 \%<\text { cloud fraction }<10 \%
\end{aligned}
$$

As will be shown in section 2c, the RH01 diagnostic is not exactly equivalent to the $\mathrm{AGCM} \mathrm{RH}_{c}$ for several reasons that are discussed in that section, but the correspondence justifies the use of RH01 as a proxy.

Data from AIRS on board NASA's Aqua spacecraft were used to compute the RH01 diagnostic. Data were obtained from the National Aeronautics and Space Administration (NASA)'s Goddard Earth Sciences Data and Information Services Center. The version 5 level 3 monthly-mean product was chosen, providing, among other fields, vertical profiles of monthly-mean cloud fraction and relative humidity using AIRS, the Advanced
Microwave Sounding Unit (AMSU), and the Humidity Sensor for Brazil (HSB) radiances for the retrieval (Aumann et al. 2003). The retrieval algorithm is described and evaluated by Susskind et al. (2011). Data are available on a $1^{\circ} \times 1^{\circ}$ grid at 12 pressure levels, and the data from 2002 to 2010 were used for this study. The monthly-mean product was chosen because of the more complete sampling in the vertical of the distribution of cloudy pixels and because of the need here for vertical profiles of relative humidity and cloud cover that could be used together.

The cloud cover estimates were evaluated by Stubenrauch et al. (2008) and compared to estimates from instrumentation on the Cloud-Aerosol Lidar and Infrared Pathfinder Satellite Observations (CALIPSO) satellite. The authors found good agreement between the AIRS and CALIPSO cloud height and cloud amounts. The water vapor estimates were evaluated by Divakarla et al. (2006), who found good agreement with rawinsonde estimates for clear-sky conditions over the ocean, and larger errors over land and for cloudy conditions. Fetzer et al. (2006) evaluated the AIRS total precipitable water and compared values to estimates from the Advanced Microwave Scanning Radiometer for Earth Observing System (AMSR-E) instrument on NASA's Aqua satellite. The authors found that the moisture difference (attributed to sampling errors in the AIRS data) can be as large as $60 \%$ when cloud fractions are large, and that the sign and magnitude of the differences depend on the geographic location and on the cloud amount. Water vapor and temperature profiles were evaluated by Susskind et al. (2006), who also found that the AIRS estimates were more accurate under conditions of reduced cloud amount.

The AIRS monthly-mean relative humidity and cloud fraction fields were used to compute the RH01 diagnostic, defined here as the relative humidity of grid boxes sampled when the cloud fraction exceeds $0 \%$ but is less than $10 \%$. The upper threshold of $10 \%$ cloud fraction was chosen to provide a large sample of grid boxes while not affecting the resulting profiles. Upper thresholds of cloud fraction between $1 \%$ and $10 \%$ were evaluated (not shown here), and the resulting profiles showed little impact of this choice. The findings of Fetzer et al. (2006) and Susskind et al. (2006) indicate that the errors in AIRS-generated relative humidity are smallest where the cloud cover is smallest, so the RH01 diagnostic is computed using the most reliable subset of the AIRS-derived relative humidity profiles.

\section{c. Suitability of RHO1 as a proxy for critical relative humidity}

The RH01 diagnostic computed from AIRS data is meant as a proxy for the AGCM critical relative humidity (or second moment of a total water PDF). The 


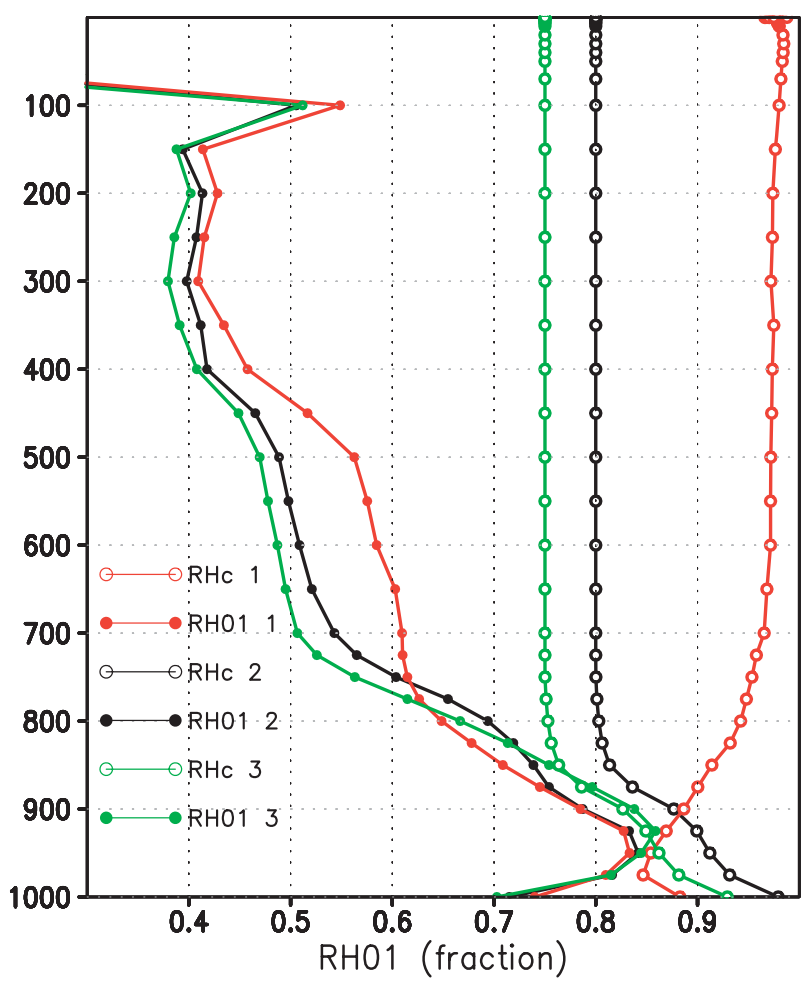

FIG. 2. RH01 diagnostic and corresponding critical relative humidity profiles from GEOS-5 simulations. The red curves are the critical relative humidity (open circle) and RH01 diagnostic (closed circle) from the profile specified in the GCM used as part of MERRA, and the black and green pairs are the critical relative humidity (open circle) and RH01 diagnostic (closed circle) from two different profiles with an hyperbolic tangent structure.

correspondence between $\mathrm{RH}_{c}$ and $\mathrm{RH} 01$ is shown in Fig. 2 with results from a series of simulations at a horizontal resolution of $2^{\circ} \times 2.5^{\circ}$ using the Goddard Earth Observing System, version 5 (GEOS-5), AGCM (Rienecker et al. 2008). The choice of $\mathrm{RH}_{c}$ profiles for this comparison includes the profiles from the AGCM used as part of the Modern Era Retrospective-Analysis for Research and Applications (MERRA) reanalysis and two profiles considered for inclusion in the Fortuna version of the GEOS-5 AGCM (Molod et al. 2012). The rationale for the different AGCM-specified $\mathrm{RH}_{c}$ profiles will be described in section 4 . The black, green, and red lines in Fig. 2 with the open circle markers represent the three different $\mathrm{RH}_{c}$ profiles from three different experiments, referred to in the figure legend as experiments 1-3. The red line shows a typical $\mathrm{RH}_{c}$ profile from the version of the GEOS-5 AGCM used as part of the MERRA reanalysis, and the green and black lines are two examples of hyperbolic tangent-shaped $\mathrm{RH}_{c}$ profiles that were considered for use in the Fortuna version of the GEOS-5 AGCM. The three lines with the filled circle markers are the monthly-mean, global-mean RH01 diagnostic profiles computed from the simulated relative humidity and cloud cover, color coded to match the AGCM-specified $\mathrm{RH}_{c}$ profiles.

Figure 2 shows a clear correspondence between the specified $\mathrm{RH}_{c}$ profile and its resulting $\mathrm{RH} 01$ diagnostic above $750 \mathrm{mb}$. The highest $\mathrm{RH}_{c}$ corresponds to the wettest RH01 (the pair of red curves) and the lowest $\mathrm{RH}_{c}$ to the driest RH01 (the green pair of curves). The general vertical profile of $\mathrm{RH}_{c}$ is also reflected in the general shape of the RH01 curves. RH01 profiles are generally drier than the $\mathrm{RH}_{c}$ profiles, which reflects the contribution of other atmospheric processes, such as dynamical subsidence in the descending branches of the Hadley circulation. The correspondence between $\mathrm{RH}_{c}$ and RH01 is less clear in the planetary boundary layer region, where the relative humidity is determined by the interaction of the moist processes and other physical processes, such as the turbulent transfer of moisture. An example of this type of environment would be the eastern Pacific, where the balance between surface turbulent exchange and large-scale atmospheric subsidence governs the behavior. In this type of regime, $\mathrm{RH}_{c}$ does not exert the sole control over the gridbox relative humidity. The existence of environments with long-lived clouds that are characterized by small amounts of condensate, which would be sampled as part of RH01, could also account for some of the discrepancy between $\mathrm{RH}_{c}$ and $\mathrm{RH} 01$ profiles.

\section{d. The use of monthly-mean AIRS fields}

The use of the monthly-mean AIRS estimates of cloud cover and relative humidity allows adequate sampling of AIRS cloudy soundings for the examination of full vertical profiles (Aumann et al. 2003). The retrieval from an instantaneous AIRS sounding can distinguish up to two levels of cloud cover where it occurs, and it is the accumulation of a month of soundings that allows the more complete vertical profile of cloud fraction to be examined. The AGCM total water PDF parameters, however, must be known at each model time step. The use of monthly-mean fields from AIRS therefore warrants an examination of the impact of the temporal averaging on the resulting RH01 profiles.

A comparison between RH01 computed from 6hourly and monthly-mean cloud and humidity fields cannot be performed using AIRS level 3 data, but it can be performed using reanalysis data. Data from MERRA, produced by NASA's Global Modeling and Assimilation Office (Rienecker et al. 2008) were used to compare the RH01 diagnostic computed from monthly-mean and 6-hourly data as input. Data from January 2003 were obtained from the NASA Goddard Earth Sciences Data and Information Services Center. The data assimilation process of MERRA included AIRS radiances during this time frame. 

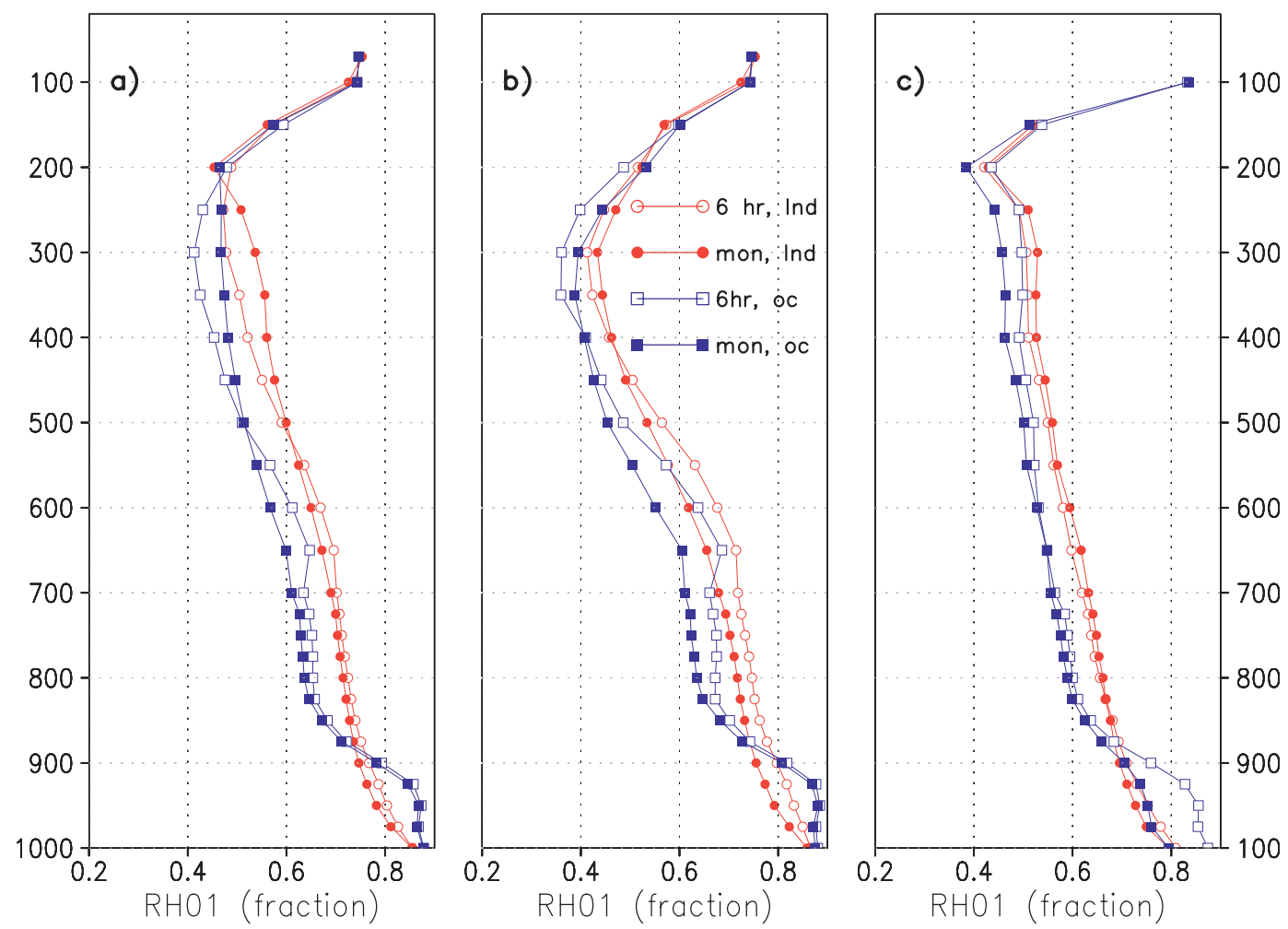

FIG. 3. RH01 diagnostic from MERRA, computed using 6-hourly (open circle and square) and monthly-mean (filled circle and square) cloud cover and relative humidity. Blue curves are for ocean averages and red for land. (a) Globally averaged RH01, (b) tropical average, and (c) extratropical average.

Figure 3 shows RH01 profiles averaged over global, tropical $\left(30^{\circ} \mathrm{S}-30^{\circ} \mathrm{N}\right)$, and extratropical $\left(30^{\circ}-60^{\circ} \mathrm{N} / \mathrm{S}\right)$ regions, separately for land (red curves) and ocean (blue curves), computed from 6-hourly (open circles and squares) and from monthly-mean (filled circles and squares) MERRA cloud and humidity fields. The comparison indicates that the choice of monthly-mean fields to calculate the RH01 diagnostic instead of means over shorter periods has a small impact. The main discrepancy is over extratropical ocean regions near the surface (below $850 \mathrm{mb}$ ), where the RH01 computed from monthly means looks more like the land profiles in all the regions and less like the oceanic profiles. There are also some discrepancies of up to $10 \%$ of relative humidity in the tropics related to the value of the RH01 above the boundary layer near $700 \mathrm{mb}$. This examination of data from MERRA is used here to assert that the use of monthly-mean AIRS estimates of clouds and humidity does not impact the resulting RH01 profiles that will be used as a proxy for the choice of the AGCM $\mathrm{RH}_{c}$.

\section{e. Behavior of AIRS RH01 diagnostic}

Figure $4 \mathrm{~b}$ shows the global-mean climatology of the AIRS-derived RH01 diagnostic for each month of the year. The global-mean profiles all show the largest RH01 values near the surface, smaller values relatively constant with height throughout the troposphere, and smaller-still RH01 values starting near $300 \mathrm{mb}$. For comparison, Fig. 4a shows the profiles of AIRS relative humidity sampled when there is no cloud cover, and Figs. $4 \mathrm{c}$ and $4 \mathrm{~d}$ show profiles of AIRS relative humidity sampled for larger cloud amounts. The sequence of profiles in Figs. 4a-d show that AIRS relative humidity values are higher when sampled during cloudier conditions, and that the shape of the vertical profile of relative humidity is different under different amounts of cloud cover. In addition, the RH01 diagnostic curves (Fig. 4b) are very similar for different months of the year, while the AIRS relative humidity sampled under different cloud amounts differ more with month. The distinct character of the RH01 profiles points to the uniqueness of the RH01 diagnostic and supports its use as a proxy for the $\mathrm{AGCM} \mathrm{RH}_{c}$.

Figure 5 shows the AIRS RH01 diagnostic in different geographical regimes and over different underlying surfaces. Figure 5a shows the global-mean RH01 separately over ocean (the group of profiles with higher RH01 values near the surface) and land surfaces for 

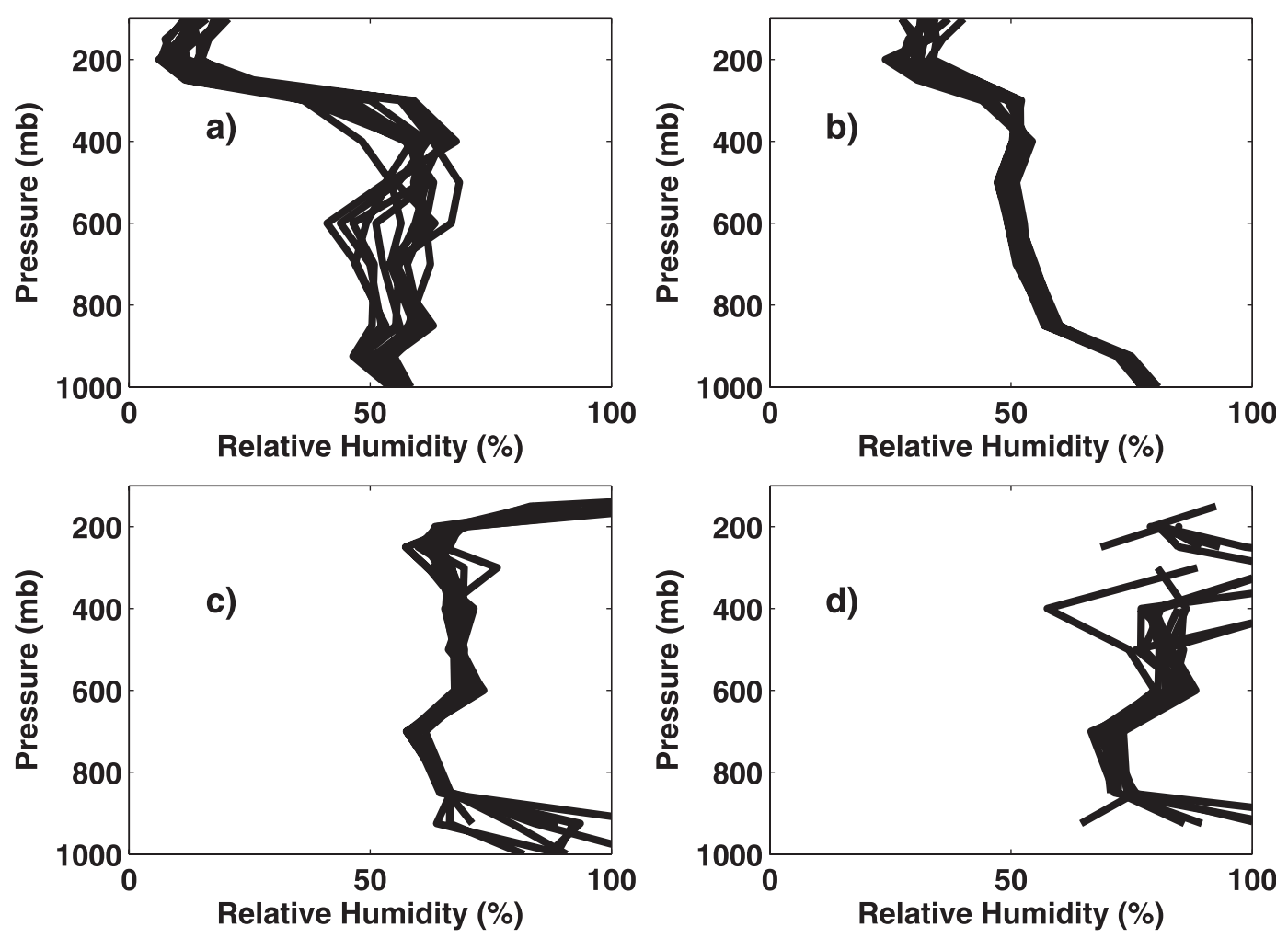

FIG. 4. (a) AIRS relative humidity fraction sampled when cloud cover is $0 \%$, and (b) RH01 diagnostic (AIRS relative humidity when cloud cover is between $0 \%$ and $10 \%$. (c),(d) AIRS relative humidity sampled when cloud cover is between $10 \%$ and $20 \%$ and between $20 \%$ and $30 \%$, respectively. Different lines represent the climatology of different months.

each month. The profiles within one group (e.g., those that correspond to ocean surfaces) are difficult to distinguish from each other, while the differences between groups are more easily distinguishable. This indicates that the month-to-month differences between RH01 profiles are small compared to differences due to the underlying surface. July values are chosen here as illustrative of the RH01 behavior. Figures $5 b-d$ show the July-mean RH01 profiles averaged over the entire globe, the tropics, and the midlatitudes, respectively, again separated into profiles over land, ocean, and the entire region. Near the surface, the largest differences between latitude bands are over the oceans, with larger RH01 values over tropical oceans. Above the boundary layer region, the RH01 profiles in the troposphere are drier in the tropics than in midlatitudes over oceans. The ocean profiles in all regions exhibit a minimum value in the midtroposphere (near $700 \mathrm{mb}$ in the extratropics and near $500 \mathrm{mb}$ in the tropics), increasing farther aloft. Above the tropopause region, RH01 values are less reliable due to the presence of water vapor mixing ratios below the AIRS sensitivity limit (Gettelman et al. 2004), and so the behavior in this region will not be examined further.
The physical interpretation of the character of the RH01 profiles described here is through its use as a proxy for $\mathrm{RH}_{c}$ and the connection between $\mathrm{RH}_{c}$ and the width of the subgrid-scale distribution of total water. The generally higher RH01 values near the surface suggest a higher $\mathrm{RH}_{c}$ and therefore a narrower PDF in the presence of boundary layer turbulence. Based on a boundary layer balance of sources and sinks of variance of total water, the results presented here imply that the net impact of boundary layer turbulence is to smooth out the subgrid variations of total water, resulting in a narrower distribution.

The AIRS-derived proxy for an AGCM $\mathrm{RH}_{c}$ resembles the behavior arrived at in many AGCMs by tuning to observed radiative fluxes. Neale et al. (2010) describes an $\mathrm{RH}_{c}$ profile that has a minimum value at $400 \mathrm{mb}$ and aloft, and increases nearer to the surface. Donner et al. (2011) describes an $\mathrm{RH}_{c}$ profile that has a minimum value at $600 \mathrm{mb}$ and increases linearly above and below to $100 \%$. The scheme of Tompkins (2002), however, shows wider PDFs near the surface, which differs from the profiles used in AGCMs and seen in the AIRS RH01 diagnostic. In the midlatitude regions, the sharp decrease of RH01 near the jet region may indicate 

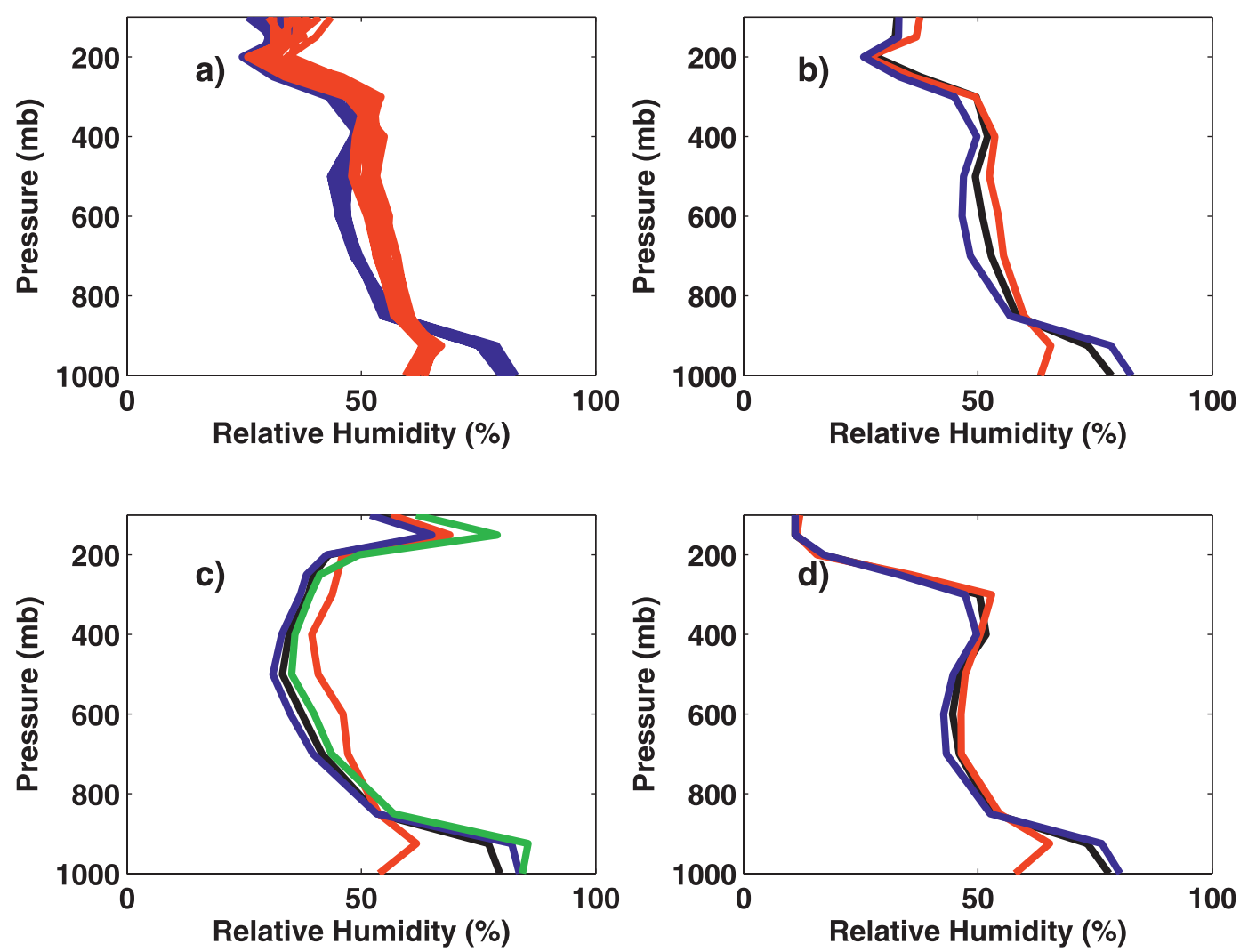

FIG. 5. RH01 diagnostic from AIRS. Blue curve is for ocean points, red is for land, and black is for all points. (a) Global average from each month of the year, repeated from Fig. 4b, but plotted separately over ocean and land points; (b) as in (a), but the July curves only; and (c) as in (b), but averaged over tropical regions. The green curve represents an average over warm tropical oceans. (d) As in (b), but averaged over extratropical regions.

a widening of the total water PDF in the presence of wind shear-generated turbulence.

\section{High-resolution model profiles of PDF second moment}

Horizontal scales of moisture variability in AGCMs are generally governed by different physical processes in different geographic regimes; however, in general, a smaller grid area would be expected to contain smaller variations. This would imply that subgrid-scale variability of total water depends on grid size, and in particular decrease as the grid size decreases. $\mathrm{RH}_{c}$ estimates, therefore, would be expected to increase as grid size decreases. A global model simulation using GEOS-5 (Rienecker et al. 2008) in its cubed sphere grid formulation at approximately $10-\mathrm{km}$ horizontal resolution and 72 vertical layers, 45 of which are in the troposphere (performed by W. Putman 2011, personal communication) is used here to examine the subgrid-scale variability of total water for several coarser-resolution grid sizes. Model results are from a boreal summertime 2005 simulation, performed using observed sea surface temperatures.

Subgrid-scale variance of total water within 200-, 100-, and $50-\mathrm{km}$ regions were computed using the $10-\mathrm{km}$ model output fields. $\mathrm{RH}_{c}$ profiles were calculated using the equations in section $2 \mathrm{a}$, defining the width of the $\mathrm{PDF}$ as twice the subgrid-scale standard deviation. Resulting $\mathrm{RH}_{c}$ profiles are shown in Fig. 6 for tropical, midlatitude, and polar regions, separately over land and ocean surfaces. The general shape of the vertical profile of the critical relative humidity is unchanged as the grid resolution changes, for all regions and underlying surfaces, and shifts to higher values with smaller grid size. This behavior is consistent with expectations.

The general shape of the high-resolution-modelderived $\mathrm{RH}_{c}$ profiles also shows higher values near the surface than in the midtroposphere, which is consistent with the AIRS RH01 profiles in Fig. 5, those in the MERRA RH01 diagnostic curves (Fig. 3), and those used in many AGCMs. This occurs despite the use in the high-resolution model simulation of the specified $\mathrm{RH}_{c}$ profile from the MERRA version of the GEOS-5 

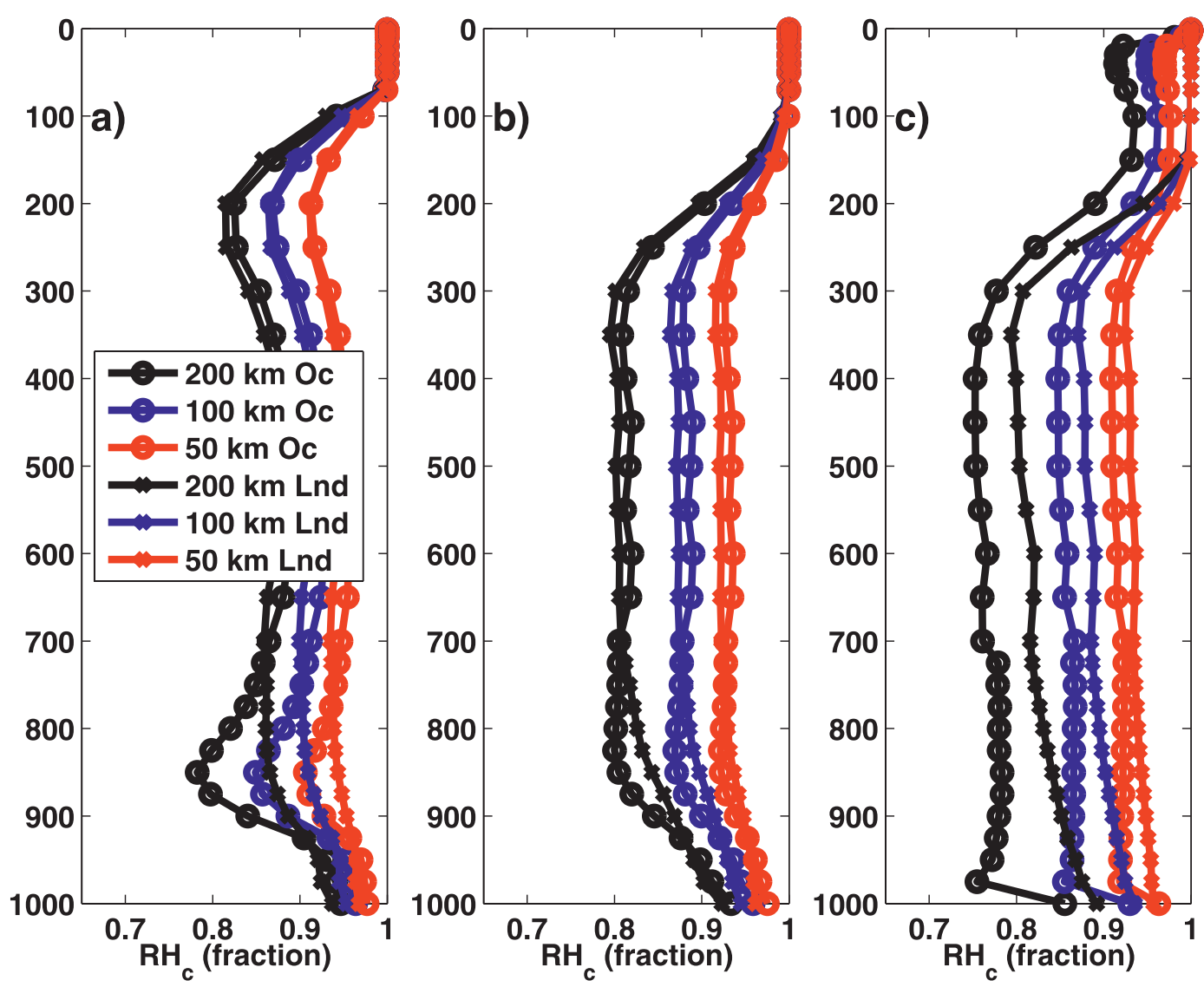

FIG. 6. July critical relative humidity profiles (fraction) at different resolutions computed from the 10-km global GEOS-5 simulation standard deviations. Black curves are for 200-km resolution, blue for $100-\mathrm{km}$ resolution, and red for 50-km resolution. Open circles are averages over ocean points; crosses are averages over land points. (a) Tropical average, (b) extratropical average, and (c) polar region average.

AGCM, shown as the red line with open circles in Fig. 2. The profiles in Fig. 6 also show a rapid increase of $\mathrm{RH}_{c}$ with height near the tropopause level in each geographical region. This is consistent with the MERRA RH01 diagnostic and the AIRS tropical RH01 profiles (though not the extratropical ones), and the profile used in the Atmospheric Model, version 3 (AM3), AGCM (Donner et al. 2011). The significance or relevance for AGCM simulations of the $\mathrm{RH}_{c}$ profile near or above the tropopause is unclear.

\section{AGCM simulations with AIRS-guided critical RH}

Three AGCM simulations with the GEOS-5 AGCM were performed to assess the impact of changes in the specified profiles of $\mathrm{RH}_{c}$. The simulations were $30-\mathrm{yr}$ duration, initialized on December 1979, at a resolution of $2^{\circ} \times 2.5^{\circ} \times 72$ levels. All experiments with the GEOS5 AGCM make use of a top hat-shaped PDF of total water. The specified profiles of $\mathrm{RH}_{c}$ represent the only difference among the three experiments, one of which, experiment 1 , uses the $\mathrm{RH}_{c}$ from the MERRA AGCM version of GEOS-5, shown as the black curve with open circles in Fig. 2, and the other two, experiments 2 and 3, use profiles chosen based on the AIRS-derived proxy, the RH01 diagnostic, shown as the black and green curves with open circles in Fig. 2. The MERRA AGCM $\mathrm{RH}_{c}$ profile was chosen using the rationale that the values are close to 1 , and are reduced by jet region shear turbulence and by boundary layer turbulence. The assumption that $\mathrm{RH}_{c}$ is reduced due to boundary layer turbulence was abandoned in experiments 2 and 3 , as it is inconsistent with what is inferred about $\mathrm{RH}_{c}$ from the AIRS RH01 profiles and the high-resolution model results, discussed in sections $2 \mathrm{e}$ and 3 .

The general shape of the RH01 profiles seen in Fig. 5 is characterized in all geographic regions over oceans by large values near the surface, a rapid drop with height below approximately $800 \mathrm{mb}$, and a relatively constant profile with height throughout the free troposphere. Over land areas the RH01 near the surface is smaller 

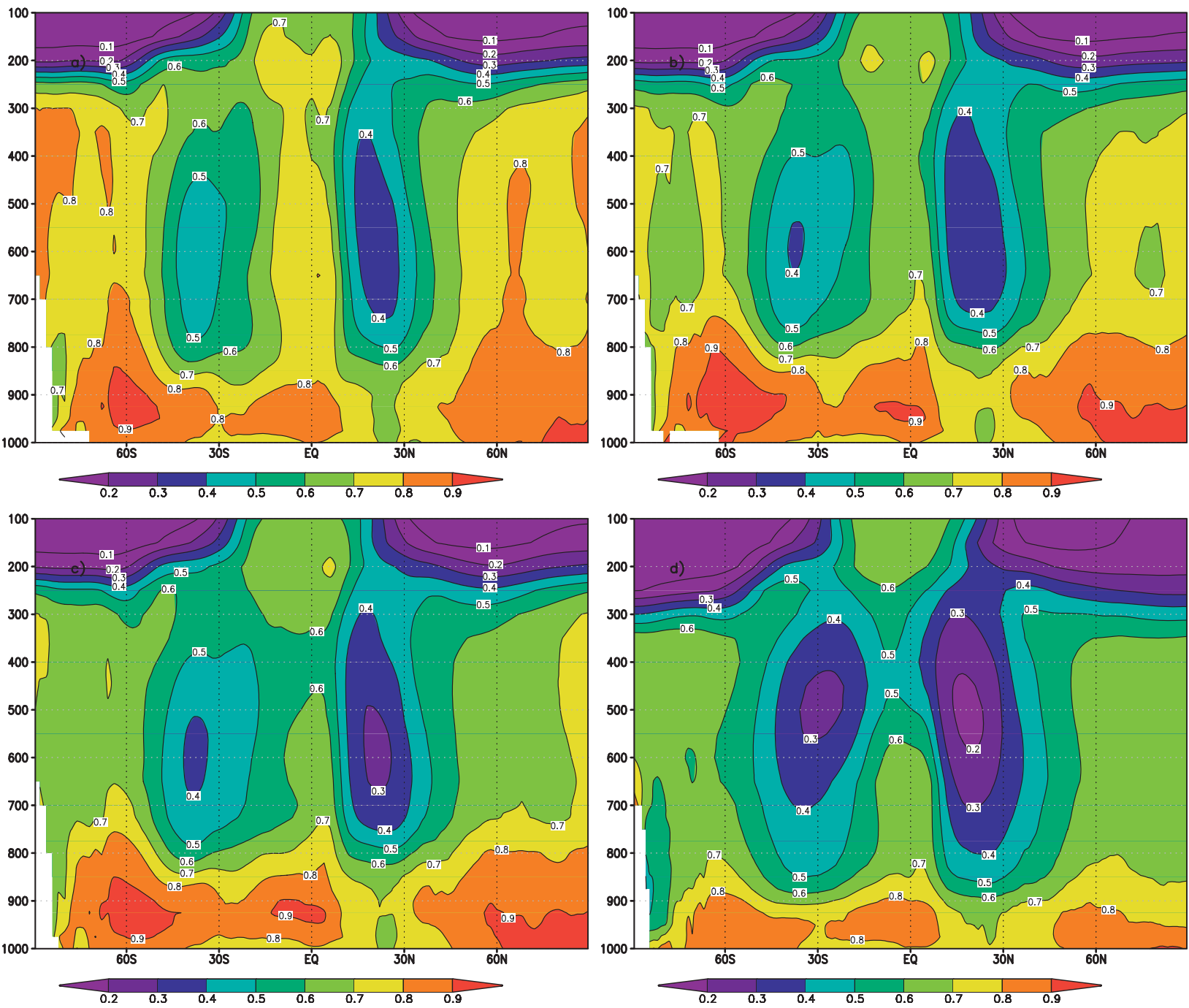

FIG. 7. Zonal-mean relative humidity in fraction for the DJF season from MERRA and from several 20-yr-long GEOS-5 GCM simulations using different profiles of critical relative humidity. (a) Simulation using the profile in the GCM used in MERRA (as in the red curve in Fig. 2), (b) simulation using the profile shown in the black curve in Fig. 2, (c) simulation using the profile shown in the green curve in Fig. 2, and (d) MERRA climatology.

than over ocean areas. These general features are characterized in the Fortuna version of the GEOS-5 AGCM by a hyperbolic tangent function, and AGCM experiments 2 and 3 described here use a hyperbolic tangent functional form for $\mathrm{RH}_{c}$ with two different sets of parameters to control the location of the hyperbolic tangent function on the $\mathrm{RH}_{c}$ axis. The AGCM-specified $\mathrm{RH}_{c}$ value near the surface over the continents is set to 0.01 plus its value in the midtroposphere. The hyperbolic tangent functional form is also present in the MERRA RH01 profiles, seen in Fig. 3, and in the high-resolution model profiles of $\mathrm{RH}_{c}$ seen in Fig. 6, most clearly in the extratropics (Fig. 6b) but in the tropics as well (Fig. 6a).

The immediate impact of a lower value of $\mathrm{RH}_{c}$ on an AGCM computation of cloud and condensate is that condensation or deposition can take place in a drier grid box and so should occur more frequently, thereby increasing cloud cover and raising relative humidity. However, the restriction of allowing evaporation or sublimation up to $\mathrm{RH}_{c}$ should cause a drier atmosphere with less cloud cover. To assess which of these competing processes determines the mean climate of the AGCM simulation, the simulated December-February (DJF) climatological relative humidity and cloud fraction fields from the different simulations are examined. Figures 7 and 8 show the cloud cover and relative humidity from experiments $1-3$, the relative humidity from MERRA, and the cloud fraction from AIRS. The AIRS cloud fraction is not an exact analog to the cloud fraction as calculated in the AGCM, but it will be used here as 

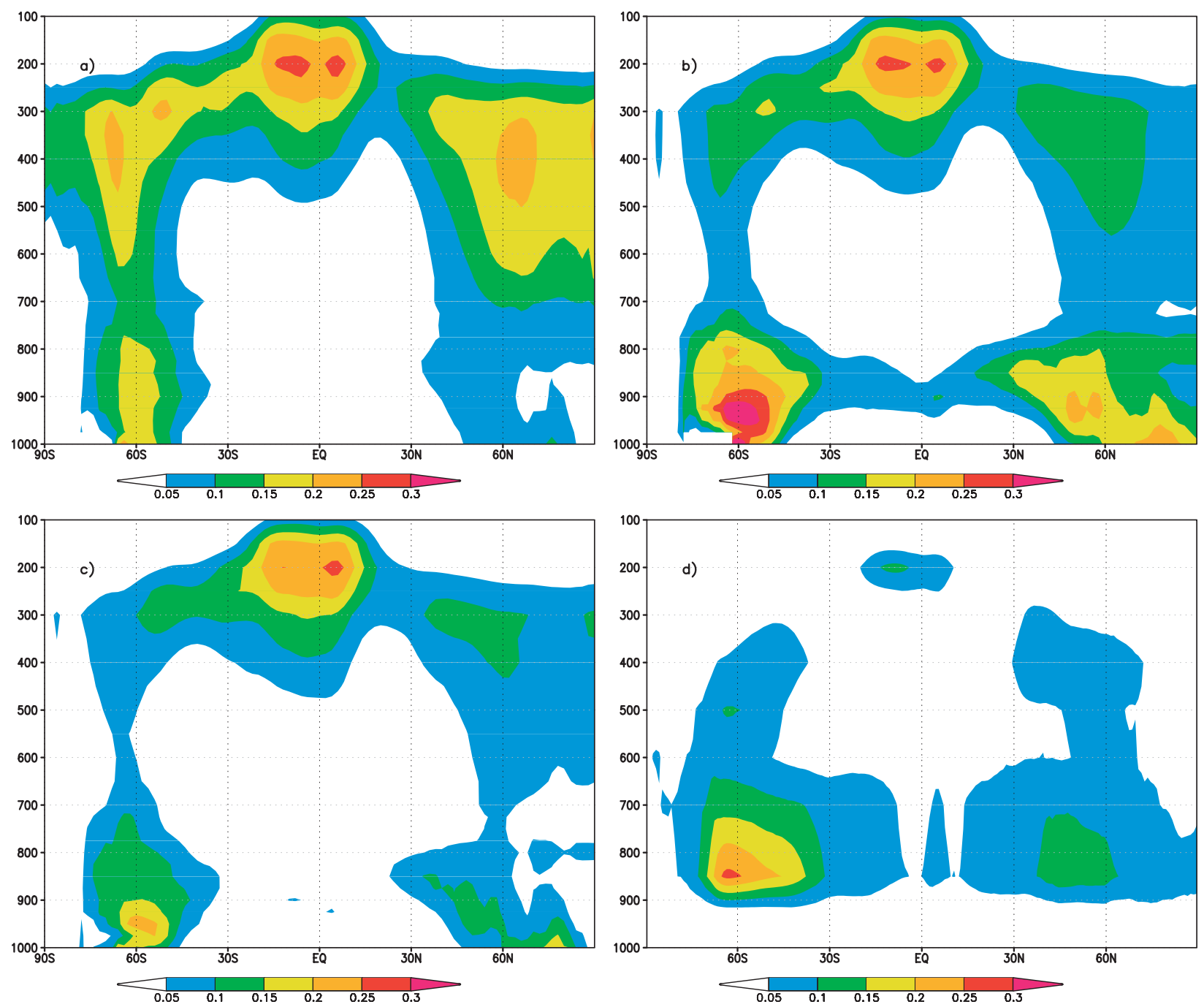

FIG. 8. Zonal-mean cloud fraction for the DJF season from AIRS and from several 20-yr-long GEOS-5 GCM simulations using different profiles of critical relative humidity. (a) Simulation using the profile in the GCM used in MERRA (as in the red curve in Fig. 2), (b) simulation using the profile shown in the black curve in Fig. 2, (c) simulation using the profile shown in the green curve in Fig. 2, and (d) AIRS climatology.

a comparison for an assessment of the general model behavior.

The impact of changing $\mathrm{RH}_{c}$ on the mean relative humidity is shown in Fig. 7. Relative humidity aloft, particularly in the extratropics, decreases with decreasing $\mathrm{RH}_{c}$, as seen in the progression from Fig. 7a (experiment 1 using the red $\mathrm{RH}_{c}$ profile in Fig. 2) to Fig. $7 \mathrm{~b}$ (experiment 2 using the black $\mathrm{RH}_{c}$ profile) to Fig. 7c (experiment 3 using the green $\mathrm{RH}_{c}$ profile). This progression reveals that it is the second of the competing processes-namely, the restriction on the evaporation and sublimation processes imposed in the model by a drier $\mathrm{RH}_{c}$ - that determines the mean climate of the AGCM simulation. The two figures with results from simulations with the AIRS-guided $\mathrm{RH}_{c}$ (Figs. $7 \mathrm{~b}$ and 7c) closely resemble each other and more closely resemble the MERRA relative humidity (Fig. 7d), although they are both still biased wet.

Figure 8 shows that cloud fractions above $500 \mathrm{mb}$, particularly in the extratropics in both hemispheres, also decrease with decreasing $\mathrm{RH}_{c}$, as seen in the progression from Figs. $8 \mathrm{a}$ to $8 \mathrm{~b}$ to $8 \mathrm{c}$. The profiles from Figs. $8 \mathrm{~b}$ and $8 \mathrm{c}$ in the extratropics aloft exhibit a structure that more closely resembles AIRS estimates, although still biased high. Near the boundary layer top, at approximately $900 \mathrm{mb}$, the trend of decreasing cloud cover with decreasing $\mathrm{RH}_{c}$ is also apparent, but in the lower part of the atmosphere the largest $\mathrm{RH}_{c}$ is from experiment 2, shown in Fig. 8b (the black $\mathrm{RH}_{c}$ profile in Fig. 2); the next in the sequence of decreasing $\mathrm{RH}_{c}$ near the surface is 
experiment 1 shown in Fig. 8a; and the smallest is experiment 3 , shown in Fig. 8c. It is the cloud fraction in experiment 2 (Fig. 8b), which most closely resembles the AIRS cloud fraction near the surface.

The AGCM simulations show, in general, that the resulting AGCM climate simulation is highly sensitive to the choice of the specified $\mathrm{RH}_{c}$ and its vertical structure. The behavior of the cloud cover and relative humidity fields, along with the assessment of the resulting climate of the atmosphere-ocean coupled simulations contributed to the choice of the $\mathrm{RH}_{c}$ profile used in experiment 2 for the Fortuna version of the GEOS-5 AGCM (Molod et al. 2012) for simulations at $2^{\circ} \times 2.5^{\circ}$ resolution. The combination of the change in $\mathrm{RH}_{c}$ between the MERRA and Fortuna versions of the GEOS-5 AGCM along with many other differences between the model versions resulted in an improved simulation relative as assessed by a comparison with many reanalysis and satellite-derived fields (Molod et al. 2012).

\section{Summary and conclusions}

AGCM cloud parameterizations include an assumption about the subgrid-scale probability distribution of total water or a related variable. The width of the subgrid-scale PDF is directly related to the AGCM critical relative humidity. AIRS monthly-mean cloud amount and relative humidity fields were used to compute a diagnostic quantity defined here that is a proxy for the second moment of an AGCM total water PDF. The AIRS-derived "RH01 diagnostic," defined as the gridbox relative humidity sampled when the cloud fraction is greater than $0 \%$ and less than $10 \%$, was shown to correspond to the AGCM critical relative humidity, and the use of monthly-mean fields rather than instantaneous fields to compute monthly-mean RH01 fields was shown not to impact the results.

The AIRS RH01 diagnostic is generally larger near the surface than aloft, and varies most markedly with the type of underlying surface. The RH01 profiles differ somewhat among geographic regions, the tropical profiles being drier aloft and wetter near the surface than extratropical profiles. The seasonal dependence is small. The dependence of the width of the AGCM total water PDF on horizontal grid resolution was shown using a high-resolution (approximately $10 \mathrm{~km}$ ) global model simulation with the GEOS-5 AGCM. High-resolution total water values were used to compute subgrid-scale variances within coarser-resolution boxes. The vertical structure of $\mathrm{RH}_{c}$ profiles computed from the variance is unchanged as the grid resolution increases from 200 to 100 to $50 \mathrm{~km}$, and the $\mathrm{RH}_{c}$ profiles shift toward higher values with increasing resolution.
Several GEOS-5 AGCM simulations of 30-yr duration were performed with the $\mathrm{RH}_{c}$ profile used as part of the MERRA version and with two choices of $\mathrm{RH}_{c}$ values specified based on the AIRS proxy. The resulting cloud and humidity fields were shown to be quite sensitive to the prescribed $\mathrm{RH}_{c}$, and improved relative to observational estimates by using the AIRS-guided profiles. The AIRS-guided $\mathrm{RH}_{c}$ profiles, including their dependence on the underlying surface type and on horizontal resolution, have been implemented in the GEOS-5 AGCM in its configuration for use in coupled and uncoupled climate simulations, data assimilation, and numerical weather prediction (Molod et al. 2012).

The AIRS-derived RH01 diagnostic was examined here in terms of regional behavior. The analysis of the localized geographic dependence will result in an empirical state-dependent formulation for the $\mathrm{AGCM} \mathrm{RH}_{c}$, and it will be implemented in a future version of the GEOS-5 AGCM. The dependence on horizontal resolution presented here will also be extended to smaller grid sizes by analyzing a simulation performed at approximately $5 \mathrm{~km}$ globally.

Acknowledgments. The author gratefully acknowledges the helpful discussions about the work presented here with Max Suarez. Interactions with J. Susskind about AIRS data were useful in choosing the correct products and gaining a sense of their reliability. The author also wishes to acknowledge W. Putman for performing the high-resolution model simulations with GEOS-5 and making them available. This research was supported by the NASA Modeling, Analysis, and Prediction program under WBS 802678.02.17.01.11. Computational support was provided by the NASA Center for Climate Simulation (and/or the NASA Advanced Supercomputing Division).

\section{REFERENCES}

Aumann, H. H., and Coauthors, 2003: AIRS/AMSU/HSB on the Aqua mission: Design, science objectives, data products, and processing systems. IEEE Trans. Geosci. Remote Sens., 41, 253-264.

Bacmeister, J. T., M. J. Suarez, and F. R. Robertson, 2006: Rain reevaporation, boundary layer-convection interactions, and Pacific rainfall patterns in an AGCM. J. Atmos. Sci., 63, 33833403.

Collins, W. J., and Coauthors, 2008: Evaluation of the HadGEM2 model. Met Office Hadley Centre Tech. Note HCTN 74, 47 pp. [Available online at http://www.metoffice.gov.uk/publications/ HCTN/index.html.]

Divakarla, M. G., C. D. Barnet, M. D. Goldberg, L. M. McMillin, E. Maddy, W. Wolf, L. Zhou, and Z. Liu, 2006: Validation of Atmospheric Infrared Sounder temperature and water vapor retrievals with matched radiosonde measurements and 
forecasts. J. Geophys. Res., 111, D09S15, doi:10.1029/ 2005JD006116.

Donner, L., and Coauthors, 2011: The dynamical core, physical parameterizations, and basic simulation characteristics of the atmospheric component AM3 of the GFDL global coupled model CM3. J. Climate, 24, 3484-3519.

Fetzer, E. J., B. H. Lambrigtsen, A. Eldering, H. H. Aumann, and M. T. Chahine, 2006: Biases in total precipitable water vapor climatologies from Atmospheric Infrared Sounder and Advanced Microwave Scanning Radiometer. J. Geophys. Res., 111, D09S16, doi:10.1029/2005JD006598.

Gettelman, A., and Coauthors, 2004: Validation of Aqua satellite data in the upper troposphere and lower stratosphere with in situ aircraft instruments. Geophys. Res. Lett., 31, L22107, doi:10.1029/2004GL020730.

Molod, A., L. Takacs, M. J. Suarez, J. Bacmeister, I.-S. Song and A. Eichmann, 2012: The GEOS-5 atmospheric general circulation model: Mean climate and development from MERRA to Fortuna. NASA Tech. Rep. Series on Global Modeling and Data Assimilation, NASA/TM-2012-104606, Vol. 28, 112 pp.

Neale, R. B., and Coauthors, 2010: Description of the NCAR Community Atmosphere Model (CAM 5.0). NCAR Tech. Note NCAR/TN-486+STR, 268 pp. [Available online at http://www. cesm.ucar.edu/models/cesm1.0/cam/docs/description/cam5_ desc.pdf.]

Norris, P., A. da Silva, and L. Oreopoulos, 2010: Using MODIS cloud observations to constrain a statistical cloud parameterization in the NASA GEOS-5 GCM. Proc. The A-Train Symp., New Orleans, LA, National Aeronautics and Space Administration, Abstract 205.

Rienecker, M. M., and Coauthors, 2008: The GEOS-5 Data Assimilation System-Documentation of versions 5.0.1 and 5.1.0, and 5.2.0. NASA Tech. Rep. Series on Global Modeling and Data Assimilation, NASA/TM-2008-104606, Vol. 27, $92 \mathrm{pp}$.
Schmidt, G. A., and Coauthors, 2006: Present-day atmospheric simulations using GISS ModelE: Comparison to in situ, satellite, and reanalysis data. J. Climate, 19, 153-192.

Slingo, J. M., and B. Ritter, 1985: Cloud prediction in the ECMWF model. ECMWF Tech. Rep. 46, 49 pp.

Smith, R. N. B., 1990: A scheme for predicting layer clouds and their water content in a general circulation model. Quart. J. Roy. Meteor. Soc., 116, 435-460.

Stubenrauch, C. J., S. Cros, N. Lamquin, R. Armante, A. Chédin, C. Crevoisier, and N. A. Scott, 2008: Cloud properties from Atmospheric Infrared Sounder and evaluation with CloudAerosol Lidar and Infrared Pathfinder Satellite Observations. J. Geophys. Res., 113, D00A10, doi:10.1029/2008JD009928.

Susskind, J., C. Barnet, J. Blaisdell, L. Iredell, F. Keita, L. Kouvaris, G. Molnar, and M. Chahine, 2006: Accuracy of geophysical parameters derived from Atmospheric Infrared Sounder/ Advanced Microwave Sounding Unit as a function of fractional cloud cover. J. Geophys. Res., 111, D09S17, doi:10.1029/ 2005JD006272.

_ perature sounding and quality control methodology using AIRS/AMSU data: The AIRS Science Team version 5 retrieval algorithm. IEEE Trans. Geosci. Remote Sens., 49, 883 907, doi:10.1109/TGRS.2010.2070508.

Tiedtke, M., 1993: Representation of clouds in large-scale models. Mon. Wea. Rev., 121, 3040-3061.

Tompkins, A., 2002: A prognostic parameterization for the subgrid-scale variability of water vapor and clouds in large-scale models and its use to diagnose cloud cover. J. Atmos. Sci., 59, 1917-1942.

Watanabe, M., S. Emori, M. Satoh, and H. Miura, 2009: A PDFbased hybrid prognostic cloud scheme for general circulation models. Climate Dyn., 33, 795-816.

Zhu, P., and P. Zuidema, 2009: On the use of PDF schemes to parameterize sub-grid clouds. Geophys. Res. Lett., 36, L05807, doi:10.1029/2008GL036817. 